\title{
Fonctions et dynamisme des jeux de miroir chez AssiaDjebar
}

\begin{abstract}
Résumé:
Dans l'extrait intitulé La femme en morceaux d'Oran, Langue morte, A.Djebar met en scène une enseignante de français qui invite ses élèves à la lecture d'un conte des Mille et une nuits. $\mathrm{Ce}$ recours au texte fondateur de la culture orientale met en place une stratégie de projection d'une culture à l'autre. Cela permet d'expérimenter l'altérité par l'immersion dans un contexte culturel, historique et social étranger.
\end{abstract}

Mots -clés : conte, interculturalité, intertextualité, contexte, imaginaire

$$
\begin{aligned}
& \text { في مقطع: "المر أة المقطعة "المقتبس من رواية } \\
& \text { "وهران لغة ميتة" اسيا جبار تقص لنا عن } \\
& \text { استاذة لغة فرنسية تطلب من تلاميذها التطلع } \\
& \text { على "قصة الف لبلة وليلة". و هذا الرجوع } \\
& \text { الى النص الاول لنقافة الثرقية يضع } \\
& \text { استراتيجية التطلع على ثقافة الاخر بيمنح } \\
& \text { تجريبة الغيرية بالغمر في سياق ثقافي } \\
& \text { ،تاريخيو اجتماعي اجنبي خيالي ،التواصل } \\
& \text { الثقافي ،الحكايات ،التتاص ،السياق. }
\end{aligned}
$$

\section{Dr.Hanène logbi}

Département des lettres et langue française Université des frères Mentouri

Constantine 1

\section{Introduction :}

Le texte littéraire porte en lui de nombreuses et diverses potentialités identifiables par une lecture qui interroge, pour cela il constitue un espace de rencontres et de médiations porteur de richesses significatives. L'illustration de ce constat puise dans un court texte d'AssiaDjebar « La femme en morceaux" extrait de son recueil intitulé «Oran, langue morte $»$. 
Le recueil constitué essentiellement de nouvelles contient pourtant un conte inspiré des Mille et une Nuits. L'écriture chezAssiaDjebar prend ainsi sa source dans ce patrimoine culturel arabo-musulman fondé sur un imaginaire bien circonscrit et s'engage dans des jeux de miroir entre le conte des Mille et une nuits et celui écrit par l'auteur. Cette forme d'écriture trace différentes pistes de réflexion.

Ce conte malgré sa brièveté peut être considéré, en effet, sous différents angles. Il interpelle, d'abord, par l'histoire narrée et sa relation à l'Histoire, puis, par les rapports d'intertextualité qu'il entretient avec le conte extrait des Mille et une Nuits, du fait de son contenu, mais aussi par la structure de l'enchâssement qui renferme l'extrait en question pris comme hypotexte, enfin, il peut être envisagé sous l'angle de la problématique de l'altérité.

L'histoire narrée est celle d'une jeune enseignante algérienne qui finit assassinée dans sa salle de cours, sous les yeux de ses élèves, alors qu'elle étudiait avec eux un conte des Mille et uneNuits. Alger 1994 sert de cadre spatio-temporel. Cette date revêt un caractère de marqueur historique. A cette époque, l'Algérie connaît un bouleversement social et politique et vit au rythme des attentats. L'on a appelé cette période de violence et de sang, «la décennie noire $»$.

\section{Relation du conte de Djebar à l'Histoire}

La littérature, traditionnellement considérée comme moyen d'évasion des contingences du réel par la voie de l'imaginaire, assure paradoxalement la perpétuation du contact avec un certain réel, souvent, en recourant aux textes venus de l'oralité. Contes, légendes et mythes fixent la mémoire collective en se référant à des représentations communes à une société donnée. Ils évoquent des situations ou des motifs qui circulent ou ont circulé dans la dite société.

Le conte étudié en classe, extrait des Mille et une Nuits, raconte l'histoire d'une femme dont le corps a été retrouvé coupé en morceaux et jeté dans le fleuve. Le sultan recherche l'auteur du crime. A la fin du texte de Djebar, l'enseignante verra des hommes entrer dans sa salle de cours pour la décapiter à son tour. L'auteur, sans conteste, fait allusion aux événements tragiques traversés durant «la décennie noire » où certaines catégories sociales, dont les enseignantes, étaient particulièrement ciblées par des actes de violence. Par ce détour, Djebar met effectivement son lecteur au contact du réel à l'aide de sa 
fiction et en ayant recours au conte étudié en classe. Le choix opéré par Djebar n'est pas fortuit, il est déterminé par le contenu du texte étudié et par sa fonction de relation avec l'Histoire.

\section{Récriture ${ }^{(1)}$ du conte des Mille et une Nuits}

L'auteur d'Oran, langue morte reste fidèle aux principes d'écriture des contes des Mille et uneNuits, dont celui de l'enchâssement, pour commencer. C'est le récit de Djebar, défini par elle-même comme un conte, qui enchâsse celui extrait des Mille et une Nuits. De la même manière, l'auteur reste fidèle à la motivation première de la narration faite par Shéhérazade. Rappelons que le sultan Shahriar, trompé par sa femme infidèle, prend chaque jour une nouvelle épouse et la tue à l'aube. La fille du vizir, Shéhérazade, nouvelle épouse du sultan, pour mettre fin à cette injustice, déroule des contes durant la nuit pour distraire son époux. Elle entame un nouveau conte à chaque aube, le sultan, curieux d'en connaitre la fin, remet au lendemain la mise à mort de la conteuse. Shéhérazade raconte pour sauver sa vie et celle d'autres éventuelles futures épouses. De même Atyka, l'enseignante, se sachant menacée de mort, continue à diffuser le savoir, en racontant l'histoire de Lafemme en morceaux comme pour exorciser le sort. "Dès lors qu'un conte est assimilé à une parabole ou une fable, ce qui importe ce n'est pas le plaisir qu'il donne... c'est la leçon de la ressemblance qu'il offre avec la réalité. $\rangle^{(2)}$

Loin d'être aléatoire, l'histoire d'Atyka inspirée de celle du conte de Shéhérazade reprend et répète un motif récurrent dans l'histoire de l'humanité ayant trait à la condition féminine.

Cependant le conte n'est pas restitué par Djebar tel qu'il est rendu dans les versions les plus courantes. L'hypotexte a été récrit par elle. "La récriture se différencie de l'intertextualité (qui est plus de l'ordre de la référence) par la variation, l'intentionnalité de l'auteur et une certaine ampleur. " "Ainsi elle est plus caractérisée par la répétition que par la référence. ${ }^{(3)}$

Le conte de Djebar porte de sensibles modifications ainsi que nous allons le vérifier. Dans ce sens, il relève plus d'un pastiche que de l'intertextualité.

Sans revenir sur les débats concernant les versions édulcorées ou non, traduites dans les langues européennes, nous nous sommes référée à la version de René R. Khawam ${ }^{(4)}$, puis à celle de Jamel Eddine Beincheikh en 
collaboration avec André Miquel ${ }^{(5)}$. Deux versions que nous avons confrontées à celle de Djebar.

Pour ce qui est des titres, AssiaDjebar intitule son conte «La femme en morceaux ». Chez René R.Khawam, il s'agit de «La femme coupée », de plus, le texte de Khawam contient un intertitre "Histoire de la pomme». Quant aux deux derniers auteurs, Bencheikh et Miquel, ils optent pour « Conte des trois pommes » conté dans la 19ème nuit. En résumé, chez Khawam le titre cible l'acte de découper l'être humain, la femme, tout comme chez AssiaDjebar. Par contre, l'intertitre met en évidence l'objet désiré par celle-ci, le fruit. En revanche, chez Bencheikh et Miquel le titre est édulcoré car seules sont considérées les trois pommes. AssiaDjebar replace la focalisation sur l'acte de découper en intitulant son texte «La femme en morceaux» bien que sa version s'attarde sur la recherche de l'objet désiré, la quête des pommes. Ce qui dénote un souci de féminisme.

Il faut signaler d'autres modifications apportées par Djebar. D'une part, elle supprime le poème déclamé par le pêcheur devant le calife. Ce dernier en est ému au point qu'il donne une pièce au pêcheur en lui enjoignant de tenter une autre fois de pêcher. Ce qui a pour résultat d'extraire du fleuve la fameuse caisse contenant le corps de la femme coupée. D'autre part, AssiaDjebar s'attarde sur le voyage à Bassora du mari pour se procurer les pommes désirées pas sa femme. Ce voyage, traité en un paragraphe chez Khawam, l'est en deux phrases chez Bencheikh et Miquel, se déroule sur plusieurs pages chez Djebar. En outre, celle-ci innove en attribuant la crainte d'une quatrième grossesse au mal étrange qui ronge l'épouse. Celle-ci demande alors à son époux une pomme comme pour se faire désirer. Enfin, dernière modification chez Djebar, l'esclave noir, voleur de la pomme procurée avec difficulté et rapportée par le mari, fait l'objet d'une longue description, attribuant une allure efféminée par cet esclave. Elle donne un cachet féministe par ces modifications.

Sous la plume de Djebar, le conte est transformé tout en restant respectueux du fil narratif et des détails propres au style des « Nuits ». Ainsi sont fidèlement rapportés les éléments dans lesquels le corps de la femme coupée est retrouvé, reprenant dans l'ordre les détails du conte tels qu'ils figurent dans les différentes versions «...le voile...un voile blanc de citadine, un voile à peine entaché...A peine ensanglanté, le tapis de soie et de fil d'or...la couffe faite de feuilles de palmier...cousue soigneusement...de fil de laine rouge...cousue vigoureusement » et enfin "la caisse de bois d'olivier. ${ }^{(6)}$ 
Plus loin dans le texte, à la mort d'Atyka, ces mêmes éléments sont repris «le corps et la tête d'Atyka dans deux voiles de lin à peine entaché, ensanglanté. Les deux voiles avec leur contenu, mis à l'abri dans deux couffes, deux couffes faites de feuilles sans doute récemment coupées...cousues de fil de laine. De la laine rouge de bonne qualité. Seront cousues vigoureusement, les deux couffes seront placées à l'intérieur d'une caisse de bois d'olivier. " "(7)

Pour ce qui est des caisses, celle pour la femme tuée par son mari est :

«Une caisse scellée. Une caisse lourde à la serrure ouvragée. Achetée chez le meilleur artisan des souks de la ville. ${ }^{(8)}$

Pour celle d'Atyka, il s'agit de :

"Une caisse lourde à la serrure ouvragée. Achetée chez le meilleur artisan de la Casbah. "(9)

La symétrie est évidente, mais l'acte de récriture tout en restant fidèle à l'hypotexte apporte la touche féministe. Le conte est de la sorte adapté à l'air du temps, au vingt et unième siècle, dans la mesure où il est traversé par diverses idéologies (féminisme, postmodernisme) et ce, pour mieux se conformer à la réalité actuelle.

Ainsi, la présence de l'Histoire récente, ayant pour cadre spatio-temporel la classe d'Atyka tout en étant diluée dans les contes des Mille et une Nuits, demeure un référent puissant.

Shéhérazade avait entrepris de divertir le sultan pour sauver sa vie. Au terme de sa narration, elle aura eu trois enfants, ce qui lui vaudra la clémence du sultan. Par contre, Atyka fait le parcours inverse. Elle ne tend pas à divertir mais à instruire. Elle n'aura, malheureusement, pas la vie sauve comme Shéhérazade, bien au contraire, elle ne peut échapper aux menaces qui pèsent sur elle, du fait de son métier. La clôture de ce conte impose, en effet, une constatation, nous avons quitté la fiction pour la dure réalité d'Alger en 1994. Car on ne saurait passer sous silence le fait que l'auteur situe le conte dans une époque où les enseignantes se mettaient en danger de mort en accomplissant leur tâche.

Mais au-delà des réalités historiques, ce qui est raconté met donc toujours en scène la parole contre l'injustice faite aux femmes. L'enjeu de la prise de parole consiste à repousser la mort, à faire durer la vie. Le réel reflète, répète le conte. Il y a effet de miroir. La littérature en récupérant un fragment de la mémoire collective est traversée par un symbolisme destiné à attirer l'attention sur la condition féminine. Le caractère engagé de cette littérature se révèle à travers ce symbolisme. 
En outre, si l'imaginaire, chez Djebar, remonte le temps, pour superposer la réalité et le mythe dans une même totalité c'est pour mieux s'inscrire dans une postmodernité telle que conçue par Baudrillard. Réel et virtuel, réalité et fiction se confondent à un point tel que l'on ne sait plus où commence la réalité ni où finit la fiction et ce, par un processus de récriture et d'intertextualité. Or pour beaucoup, «l'intertextualité est une condition nécessaire à l'interculturalité. \rangle$^{(10)}$

Nous allons nous pencher maintenant sur la dimension culturelle et interculturelle du conte écrit par Djebar.

\section{Perception de l'identité/altérité: la dimension interculturelle}

La dimension interculturelle est définie comme «une dialectique complexe d'affirmation de soi et de reconnaissance d'autrui »par Zarate ${ }^{(11)}$. Ces concepts mettent en avant les idées de partage et d'échange, un croisement des regards sur les usages, les valeurs et les représentations de chaque communauté. L'interculturalité encourage l'ouverture d'esprit et suppose la coexistence d'univers culturels différents propres à la réflexion sur le »qui suisje ? », « qui est-il ? ».

Rendre compte du tragique d'un réel par le recours aux Mille et une Nuits répond à diverses exigences. Le conte est choisi comme support matériel pour enseigner une langue étrangère (le français) dans le récit cadre. N'oublions pas que dans son parcours personnel, AssiaDjebar a vécu des expériences d'animation de classes de langue (aux États-Unis notamment), cette expérience est mise à profit dans son projet d'écriture littéraire. Dès lors, il est possible de concevoir aisément ce texte comme document didactique.

Une des réflexions qui traduit le souci de l'enseignement des langues est cette réplique d'Atyka «mais vous verrez avec des élèves vraiment bilingues, le français me servira pour aller et venir dans tous les espaces autant que dans plusieurs langues. $\rangle^{(12)}$

En classe, elle permet à ses élèves de lire le texte dans les deux langues, l'arabe et le français: "Nous serons demain à Bassora! Regardez une carte...apportez le texte - le texte arabe pour ceux qui veulent et le texte traduit. ${ }^{(13)}$,Illustration de l'intérêt pédagogique du texte étudié et du souci de l'enseignante. 
Ceci autorise l'ouverture sur un monde autre, pluriel. Le bilinguisme étant une voie vers la découverte de la différence et l'identification des repères culturels familiers à l'apprenant.

\subsection{Le conte: un genre approprié}

Si l'intertextualité favorise l'émergence de l'interculturalité, le conte «en traversant les frontières spatio-temporelles et culturelles devient alors un lieu de médiation. $~^{(14)}$. Le conte joue alors pleinement son rôle de passerelle entre deux langues et deux cultures. Dans ce cas, il assure la perception et la compréhension des mondes propre et Autre, similaire et dissemblable, nécessaire à l'interculturalité.

En outre, l'aspect oral n'est pas négligé par l'auteur, tantôt les apprenants lisent des passages, tantôt l'enseignante raconte durant le cours; le conte est découvert par bribes, il est découpé. D'une part, il est scindé selon les différentes séances de cours qui se chiffrent à cinq, d'autre part, les séquences de lecture sont interrompues par les apprenants qui sont encouragés à commenter. La lecture à voix haute provoque des réactions immédiates, permet de développer des discussions. Les différents événements lus ou narrés peuvent être commentés ou analysés, ils font l'objet de débats. Le choix du support favorise, donc, la mise en place d'un processus d'interaction. Les apprenants sont entrés dans le conte. Ils réfléchissent dans l'interaction, mettent en commun leurs propres valeurs et leur culture. Ils adhèrent ou pas à l'histoire au fur et à mesure qu'ils la découvrent. Ils essaient de comprendre, d'analyser les situations, d'exprimer leurs opinions, d'anticiper les événements à venir. Ils établissent ainsi, un réseau de rapports à l'intérieur du groupe ayant une identité culturelle partagée.

\subsection{Objectifs interculturels}

Le conte de «La femme en morceaux »ouvre un univers particulier pour tout lecteur, l'univers de la cour du sultan Haroun Er Rachid. Un texte tiré d' un recueil autre que celui des Mille et uneNuits aurait-il eu le même impact sur les apprenants?

Ce conte, précisément, plonge le destinataire dans un contexte géographique, historique, social et culturel lointain, apparemment. Ce qui est propice à la distanciation: l'étonnement, la curiosité des jeunes apprenants sont mis à contribution. Leur éveil est sollicité par le différent, par le nouveau. 
De fait, le regard étonné devient vite un regard réflexif. L'interculturel est fondé sur deux axes, l'un affectif et l'autre réflexif.

Du point de vue historique, il faudra remonter dans un temps reculé, celui du califat de Haroun Er Rachid dont les réalités sociales sont forcément différentes de celles des apprenants. La toute-puissance du sultan qui détient le pouvoir absolu et l'esclavagisme sont deux réalités faisant partie d'un autre monde, aujourd'hui dépassé. La problématique de l'altérité/identité porte essentiellement sur les relations socioculturelles. L'étrangeté des mœurs de la cour du sultan (droit de vie et de mort sur tous les sujets, présence de l'esclave noir Rayan), en éveillant la curiosité des apprenants, aura pour conséquence la projection sur l'axe affectif avec le rejet du différent. Mais dans un deuxième mouvement, par l'axe réflexif, il y aura la reconnaissance de soi-même, donc, de l'identité. Malgré tout, le contexte culturel arabo-musulman est là et les apprenants, étant confrontés à l'altérité, découvrent que cette altérité n'est pas tellement lointaine ni étrangère. La notion de "proximité dansle lointain », évoquée par Porcher, trouve ici tout son sens. Ainsi les apprenants sont confrontés à une altérité qui ne leur est pas complètement fermée. De fait, si l'on définit la culture comme "l'ensemble des valeurs acquises, intériorisées et qui fondent la cohésion d'un groupe », on distinguera, dès lors, «les valeurs profondes, authentiques, qui ne varient pas (croyances religieuses, morales...) des valeurs structurelles moins stables mais qui ont une durée de vie assez longue (institutions politiques, économiques...) et les valeurs conjoncturelles qui évoluent plus vite (mode vestimentaire...) »d'après Savagado ${ }^{(15)}$. Ce sont les valeurs profondes, authentiques ( telles que celles de justice, équité, de sensibilité au malheur du prochain qui font réagir le sultan quand il veut retrouver le coupable pour le punir, à titre d'exemple) qui seront à la base de cette proximité dans l'Altérité. Tandis que les valeurs structurelles et conjoncturelles (omnipotence et pouvoir absolu) provoquent la distanciation et s'ouvrent à la réflexion et à la comparaison (quelle est cette société qui permet au sultan de menacer de mort le vizir s'il ne trouve pas le coupable ? C'est une question que risquent de se poser les jeunes apprenants remettant en cause l'institution politique prévalant dans le conte). Les valeurs créent le lien qui permet de cerner l'intérêt socioculturel offert par un tel conte à l'enseignante.

Les apprenants sont engagés dans un processus de décentration/recentration par lequel le rejet du dissemblable a favorisé la distanciation pour réfléchir à soi-même. L'image de l'altérité contribue à se construire une identité , à se penser soi-même face à l'Autre, également à rapprocher les différences. Le 
débat libre, dont la souplesse qu'il induit, a l'avantage de permettre l'échange entre apprenants. En témoigne cet extrait :

«En ce sens, s'exclame vivement le premier garçon, cette fois sans se lever, pourrait-on dire que les contes des Mille et une Nuits sont des histoires politiques.

- Ou féministes, exulte sa voisine, la brunette. Shéhérazade dans le rôle du vizir, quelle révolution pour l'époque! » ${ }^{(16)}$

L'apprentissage de la langue débouche sur des savoir-faire interprétatifs permettant d'optimiser la relation entre la culture enseignée et celle de l'apprenant. Le but non avoué d'Atyka, sans doute, est de faire adhérer le groupe d'élèves à l'histoire narrée, puis de les conduire à faire un parallèle avec la violence qui anime leur propre société. Ainsi l'homme a toujours besoin du mythe pour parler du réel.

«Toute communication pose la question de l'Autre, de son statut, de l'altérité et de l'identité $\gg{ }^{(17)}$

Les « Nuits » assure le dépaysement nécessaire à l'étonnement qui fera réagir les apprenants par rapport à leur vécu. De fait, l'étonnement est considéré comme un puissant levier opératoire par Zarate ${ }^{(18)}$

Mais, la dimension essentielle de l'interculturel reste la décentration. Les spécialistes de l'interculturel recommandent de stimuler le regard et l'interrogation, puis de faire en sorte que les apprenants puissent par ces interrogations s'identifier et se reconnaître eux-mêmes. Le principe étant de se distancier pour réfléchir à soi-même. Et ce, en vue de relier ce qui semble autre, de rapprocher les différences. Le texte des Mille et une Nuits, tel qu'il est exploité par Atyka, remplit son contrat sur l'interculturalité. Il conduit les apprenants à s'interroger sur eux-mêmes en posant les questions sur l'organisation sociale prévalant dans le conte.

Par ailleurs, il convient de souligner que dans le contexte algérien, la nécessité de s'affirmer soi-même est fortement marquée. L'identité culturelle a failli être effacée par la colonisation. Le regard de l'Autre (occidental) l'a amoindrie, déformée, rendue fragile. Le processus de prise de conscience de soi et de ses propres marqueurs culturels peut-être enclenché par le biais des valeurs profondes manifestées dans le conte. Dans un processus inverse, il devient un lieu où l'on cherche à trouver le Même par-delà les différences. C'est ce mouvement de rejet et d'identification, de décentration et recentration qui peut apporter une réflexion enrichissante. Le Moi, marqué par une identité culturelle collective, réfléchit dans l'interaction, se distancie, s'interroge sur 
Soi et sur l'Autre, entreprend des comparaisons et exprime des façons culturelles d'être, de sentir.

Aussi nous vérifions les affirmations de G. Zarate lorsqu'elle annonce : «la description des pratiques culturelles périmées présente un grand intérêt pour le travail de distanciation par rapportau présent. ${ }^{(19)}$ Cette description permet, selon l'auteur, le recul par rapport aux représentations d'un groupe social, d'une façon d'être désormais perdue et oubliée mais dont les traces autorisent la restauration d'un parcours de sens, autour de pratiques actuelles. Autrement dit, chez Djebar, le conte des Mille et une Nuits serait « une mise en perspective qui pourrait aider à comprendre l'état des lieux » d'Alger en 1994. Il contient des pratiques culturelles, qui, bien qu'elles aient été désavouées et abandonnées depuis une époque reculée, refont surface dans le contexte du récit-cadre. Nous retrouvons, en effet, la même menace de mort qui pèse sur Shéhérazade et Atyka et la même tentative de repousser la mort par la parole. La littérature est culture, et le savoir littéraire peut se traduire en attitudes et comportements, en « savoir-être » et « savoir-faire».

\section{Conclusion}

Derrière la conteuse Shéhérazade se cache un autre conteur, Djafar, lui aussi tente de sauver son esclave de la mort en racontant au sultan l'histoire des deux frères NuredDine et Badr edDine. Mais que dire, alors, d'Atyka qui ne peut achever la lecture de ce dernier conte avec ses élèves ? Elle épilogue, malgré sa décapitation »cinq jours durant nous avons vécu avec Shéhérazade... "Ses derniers mots seront : »La nuit c'est chacun de nos jours, mille et un jour, ici chez nous, à... »(213)

Toute la symbolique est portée par cette clausule, qui annonce la mort de l'enseignante, introduit le surnaturel, au milieu d'une scène pourtant très réaliste. Elle donne au récit un cachet particulier similaire au mythe dans lequel s'introduit l'étrange, l'irréel pour décrire ce que l'homme ne peut expliquerl.

Par contre, en filigrane, elle met en parallèle deux époques et deux sociétés différentes. Cette tête tranchée d'enseignante, qui continue à conter, est emblématique de la parole qui ne saurait être bâillonnée, et qui témoigne pardelà la mort. Inspiré de faits réels, le récit rejoint de plain pied le mythe par cette introduction du surnaturel et gagne sa dimension hautement symbolique. Or ce qui importe dans le mythe, c'est moins le contenu que les relations logiques qui peuvent être reversées dans le réel. 
Enfin, AssiaDjebar a construit un récit où se croisent réalité et imaginaire, et qui pourrait bien, à son tour, être considéré comme document interculturel en classe de langue pour mettre en pratique le discours sur Soi et sur l'Altérité.

Étant établi que la convention générique du conte a été investie par un dynamisme nouveau dans le passage d'un contexte à un autre, il convient de souligner que le conte appelle à "une compréhension responsive active » (Bakhtine p. 282). Ce texte de Djebar ouvre à la réflexion interculturelle s'inscrivant dans un système où l'objet étudié est lié à l'identité culturelle des apprenants qui, en mobilisant leurs marqueurs culturels, peuvent comprendre et réagir face à cet objet.

Djebar, par la voix de cette enseignante, cherche à produire un effet didactique sur le lecteur, l'amener à réfléchir à son tour. L'effet- miroir joue donc également entre lecteur et apprenant.

\section{Bibliographie}

Corpus

Djebar A., 1996, Oran, langue morte, ActesSud-Léméac, Arles.

Abdallah-Pretceille M., 1999, Ethique de l'altérité. Interrogation et enjeux, Le français dans le monde, Paris.

Bakhtine M., 1984, Esthétique de la création verbale, Gallimard, Paris.

Bencheikh J.E., Miquel A., 2005, Les mille et une Nuits.Lescoeurs humains.T1, Gallimard, Paris.

Chraïbi A. (sous la dir...), 2004, Les Mille et une Nuits en partage, Sindbad Actes Sud, Arles.

Collés L. DevelotteG.Tauzer-Sabatelli, 2007, Didactique du FLE et l'interculturel, Editions EMS, Paris.

Gignoux A.C., 2005, Initiation à l'intertextualité, Ellipses, Paris.

Khawam R.R., 2013, Les Mille et une Nuits,Lescoeurs humainsT.2,Phébus Libretto, Paris.

Piégay-Gros N., 1996, Introduction à l'intertextualité, Dunod, Paris. Zarate G., 1986, Enseigner une culture étrangère, Hachette, Paris.

Zarate G., 2012 Représentations de l'étranger et didactique des langues, Didier coll. Essais, Paris. 


\section{Notes :}

(1)-Récriture ou réécriture : « désigne aussi de manière générale et vague toute reprise d'une ouvre antérieure, quelle qu'elle soit, par un texte qui l'imite, la transforme, s'y réfère, implicitement ou explicitement. » Nathalie Piégay-Gros, 1996, Introduction à l'intertextualité, Dunod, Paris , p.181.

(2)-François Flahault, La pensée des contes, 2001, Anthropos, Paris, p. 44.

(3)-Anne-Claire Gignoux, Initiation à l'intertextualité, 2005, Ellipses, Paris, p. 105 .

(4)-René R. Khawam, Les Mille et une Nuits,T.2Les coeurs inhumains, Phébus, Libretto, Paris.

(5)- J. E. Bencheikh et A. Miquel, 2005, Les Mille et une Nuits, T1 Les coeurs inhumains, Gallimard, Paris.

(6)-AssiaDjebar, 1996, Oran langue morte, Actes Sud-Léméac, Arles, p. 164

(7)-AssiaDjebarop .cit. p. 214

(8)-Assiadjebar op. cit. p.164

(9)-AssiaDjebarop.cit . p. 214

(10)-L. Collés, G. Develotte, Tauzer -Sabatelli, 2007,Didctique du FlE et l'interculturel, Editions EMS, Paris, p.70.

(11)-G ; Zarate, 1986 Enseigner une culture étrangère, Hachette, Paris, p.17.

(12)-A. Djebar,op. Cit. p. 280

(13)-A. Djebar ibidem

(14)-Bejjtit in Collés et alii, op.cit. p. 60

(15)-Savagado in Collés et alii, op. cit. p. 76

(16)-A. Djebar, Oran, Langue morte, op.cit.p.118

(17)-Abdallah-Pretdeille, 1999, Ethique de l'altérité. Interrogation et enjeux, Le français dans le monde,p.p.14-16.

(18)-G. Zarate,2012, Représentations de l'étranger et didactique des langues, Didier col. Essais, Paris, p.118

(19)-G. Zarate, 2012, op.cit., p. 39 